\title{
SATURATING ALL DEBTS USING MINIMUM NUMBER OF TRANSACTIONS
}

\author{
THINH D. NGUYEN
}

\begin{abstract}
Given a list of debts between pairs of people, we want to minimize the number of transactions needed to clear all debts.
\end{abstract}

\section{Problem statement}

We give an example to intuitively demonstrate our problem. Suppose we have five people Alice, Bob, Carol and Eve, some pairs of them have debts. In particular, Alice owes Bob 3 dollars, David owes Eve 10 dollars. We may even have opposite debts, such as Bob also owes Alice 5 dollars. All the debts are depicted in the following figure.

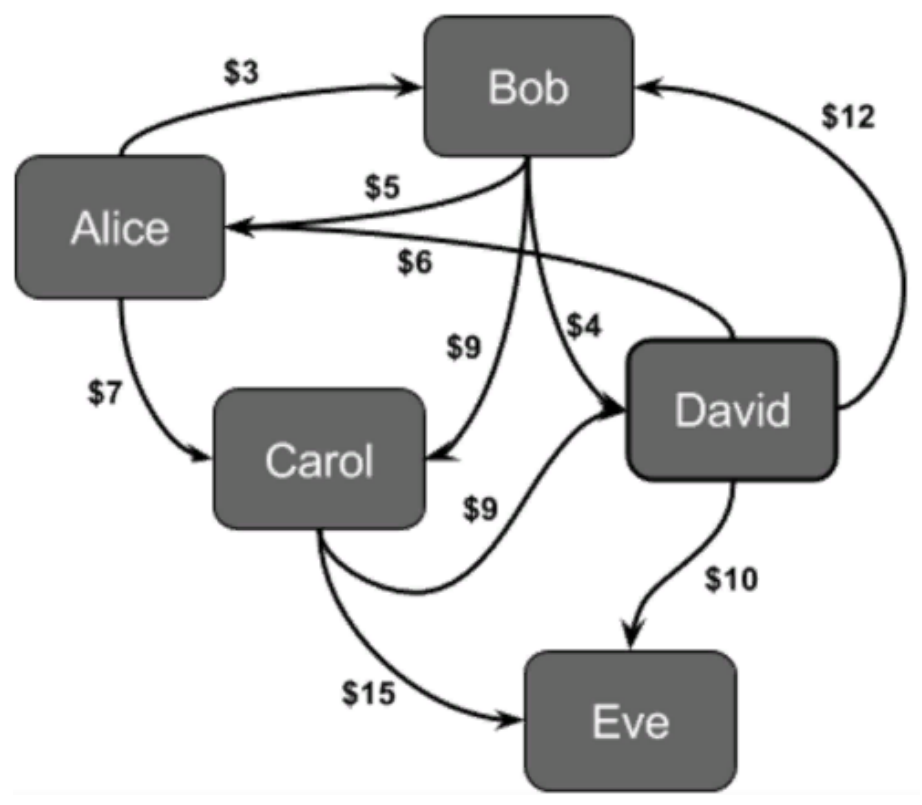

If these people want to clear all their debts, they have to make money transactions. It could be that, for each directed arc in the above graph, the two involving people make exactly the same money transaction. Clearly, doing like that requires the number of money transactions to be equal to the number of arcs in the graph. Our problem denoted by Min-Transfer asks whether $k$ money transactions are enough to saturate all the debts.

Key words and phrases. DAG, debt, network, optimization.

Perebor. 
Definition 1. (Min-Transfer problem):

Input: a directed graph $G$, a number $k$

Output: Can $k$ money transactions saturate all the debt arcs of $G$ ?

Claim 2. We have that Subset $\mathrm{Sum} \leq_{p}$ Min-Transfer

In this claim, the hardness of Subset Sum is known since [1]. Now, we prove our claim.

Proof. Our proof relies on one observation frequently seen in practice of money payments.

Some useful observations: In a minimal solution to our problem, there should not be two transactions involving three people $u, v, w$ that have $v$ as both the sender and the receiver: $u$ pays $v$ an amount of $a$ dollars and $v$ pays $w$ an amount of $b$ dollars. Clearly, having two transaction like this is annoying. If $a>b$ then $u$ needs to pay $v$ only $a-b$ dollars, and then $u$ still has the duty to pay $b$ dollars to $w$. If $a \leq b$ then $u$ directly pays $w$ an amount of $a$ dollars, and $v$ only pays $w$ the remaining amount of $b-a$ dollars. Obviously, this modification never increases the number of money transactions while still saturating the debts.

From the above observation, we can see that it is without loss of generality to assume that an optimal solution is a directed bipartite graph $(X, Y)$ in which arcs always goes from $X$ to $Y$. This is the only way to have no needed modification as before. If any vertex $v$ has some arcs going in then there is no arc going out of it.

Reduction from Subset Sum: So, we are given a set $S$ of integers and a target sum $s$. We have to decide if there exists a subset of $S$ summing to $s$. This reduction will use the positive variant of the subset problem, where all elements of $S$ and the target integer $s$ are positive.

This is the reduction:

(1) Transform the set of integers $S$ into participants who owe debt of their respective integer values.

(2) Add a "proxy" participant $c$ (short for "collector")whom each previously added participant in item 1 owe his debt

(3) Add two participants who are owed debts of $s$ and $t$ by $c$, where $t$ is the sum of all integers in the set $S$ minus $s$. This ensures that the sum of debts and sum of money owed are balanced. Call the obtained digraph $G$.

(4) Set $k$ to $n$ the cardinality of $S$. The Min-Transfer instance is $\langle G, n\rangle$

Correctness of the reduction: Clearly, $c$ has the net income as 0 and therefore does not appear in the optimal solution, i.e. $c \notin(X \cup Y)$. Each one of the participants added in item 1 has to have at least one outgoing arcs since he owes some amount at last deed. As a result, any optimal solution to our instance needs at least $n$ transactions.

If the Subset Sum is a Yes instance, we have a subset $S^{\prime} \subseteq S$ summing to $s$. So, each participant in $S^{\prime}$ directly pay their debt to $s$, bypassing "collector" $c$. The rest of the participant who owes will pay their debt to $t$. So, our Min-Transfer is also a YES instance.

Conversely, if $n$ transactions are enough to saturate our instance. Each owing participant has to pay either $s$ or $t$ but not both, since having a single participant pays both means the number of transactions is at least $n+1$. And, the owed $s$ is satisfied only when does he receive exactly his amount of money. The set of participants paying $s$ comprise the solution to the SubSET Sum instance. 


\section{Conclusion}

As long as we study a mathematical conjecture, we should encourage ourselves of having enough labouring hours on popular maths books. Then, reading some articles on theory of computing like [3] is a good practice. Only after that, could we think of the ultimate final for all mathematics sciences.

\section{REFERENCES}

1. Michael R. Garey, David S. Johnson, Computers and Intractability: A Guide to the Theory of NP-Completeness

2. David S. Johnson, The NP-Completeness Column: An Ongoing Guide. pp.393-405

3. Phan Dinh Dieu, Le Cong Thanh, Le Tuan Hoa, Average Polyno-mial Time Complexity of Some NP-Complete Problems, Theor. Comput. Sci. 46(3): pp.219-237 (1986)

Current address: Department of Mathematics, Moscow State University

Email address: kosmofarmer@yandex.com 